\title{
ORAL MUCOSITIS-RELATED NEUROPATHIC PAIN IN HEAD AND NECK CANCER PATIENTS RECEIVING RADIOTHERAPY OR CHEMORADIOTHERAPY
}

HELLENIC REPUBLIC UNIVERSITY OF ATHENS

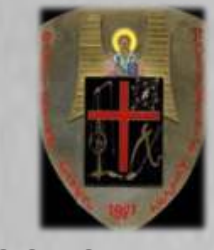

SCHOOL OF DENTISTRY CLINIC OF HOSPITAL DENTISTRY Head Professor: Head Professor:
Prof. OURANIA NICOLATOU-GALITIS
Maria Kouri $^{1}$, Ourania Nicolatou-Galitis ${ }^{1}$, Athina Vadalouca ${ }^{2}$, Vasilios Kouloulias ${ }^{3}$, Efthymios Kyrodimos ${ }^{4}$, Erofili Papadopoulou ${ }^{1}$, Emmanouil Vardas ${ }^{1}$, Evangelos Galitis ${ }^{5}$ Miltiadis Trichas ${ }^{6}$, Anna Zigogianni ${ }^{7}$, Zoi Liakouli ${ }^{7}$, Amanda Psyrri ${ }^{8}$, Ioannis Kouvaris ${ }^{7}$

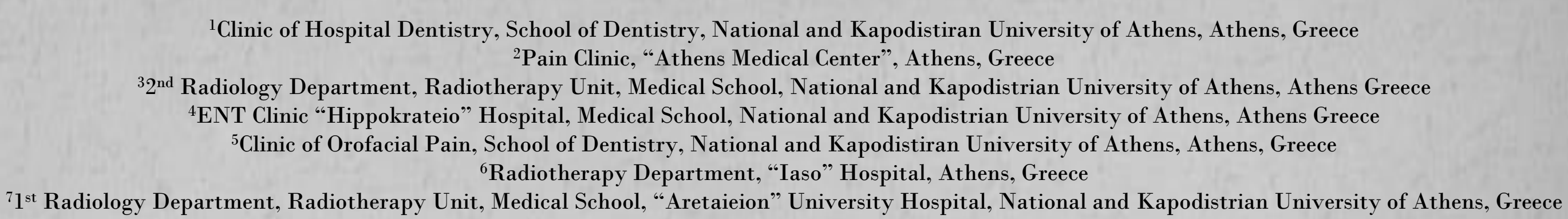

\section{INTRODUCTION:}

Painful oral mucositis (OM) is a debilitating complication in Head and Neck Cancer (HNC) patients receiving RadioTherapy (RT) /ChemoRadioTherapy. Therapy-induced pain can be nociceptive and / or neuropathic. Neuropathic pain (NP) in HNC patients often remains underdiagnosed and undertreated. This study's purpose was to identify the OM-induced NP.

\section{RESULTS:}

\begin{tabular}{|l|l|l|}
\hline Variable & N (\%) & Mean (SD) \\
\hline Age (years) & & $63.54(13.96)$ \\
\hline Gender & $20(76.9)$ & \\
Male & $6(23.1)$ & \\
Female & $2(7.7)$ & \\
\hline Cancer Stage & $7(26.9)$ & \\
II & $9(34.6)$ & \\
III & $2(7.7)$ & \\
IV & $6(23.1)$ & \\
Recurrence & & \\
missing & $16(61.5)$ & \\
\hline Cancer Site & $3(11.5)$ & \\
Oral / Oropharyngeal & $3(11.5)$ & \\
Nasal / Nasopharyngeal & $2(7.7)$ & \\
Parotid & $2(7.7)$ & \\
Unknown Primary & & \\
Other & $11(42.3)$ & \\
\hline Type of Treatment & $15(57.7)$ & \\
Radical RT & $20(76.9)$ & \\
Postoperative RT & & \\
Concomitant Chemotherapy & & $62.11(9.243)$ \\
\hline Radiotherapy Dose (Gray) & & \\
Total Dose & & \\
Daily Dose & $3(11.54)$ & \\
\hline Infections (before the DN4 completion) & \\
Candidiasis & $2(7.69)$ & \\
Herpes & $8(30.77)$ & \\
\hline Pain medication & $7(26.92)$ & \\
Opioids & $1(3.85)$ & \\
NSADs & Adjuvant (Pregabalin) & \\
\hline
\end{tabular}

Characteristics of the patients who completed a DN4q (n=26)

\begin{tabular}{|l|l|l|l|l|}
\hline & DN4 & Pain & Oral Mucositis & Xerostomia \\
\hline DN4 & & $\mathrm{r}=0.617$ & $\mathrm{r}=0.752$ & $\mathrm{r}=0.590$ \\
& & $\mathrm{p}=0.001$ & $\mathrm{p}<0.001$ & $\mathrm{p}=0.002$ \\
\hline Pain & $\mathrm{r}=0.617$ & & $\mathrm{r}=0.314$ & $\mathrm{r}=0.733$ \\
& $\mathrm{p}=0.001$ & & $\mathrm{p}=0.119$ & $\mathrm{p}<0.001$ \\
\hline Oral Mucositis & $\mathrm{r}=0.752$ & $\mathrm{r}=0.314$ & & $\mathrm{r}=0.444$ \\
& $\mathrm{p}<0.001$ & $\mathrm{p}=0.119$ & & $\mathrm{p}=0.023$ \\
\hline Xerostomia & $\mathrm{r}=0.590$ & $\mathrm{r}=0.733$ & $\mathrm{r}=0.444$ & \\
& $\mathrm{p}=0.002$ & $\mathrm{p}<0.001$ & $\mathrm{p}=0.023$ & \\
\hline
\end{tabular}

Correlations between DN4 score, intensity of pain, oral mucositis and xerostomia. (Spearmann's rho test $-r$ ).

\section{PATIENTS \& METHODS:}

ORAL PAIN AND XEROSTOMIA EVALUATION: Numeric Rating Scale (NRS); Scores: $0-10$

1-4: mild, 5-7: moderate, $8-10$ : severe.

NEUROPATHIC PAIN EVALUATION: Douleur Neuropathique 4 questionnaire: DN4; Cut-off value: total score of 4/10.

ORAL MUCOSITIS GRADING: EORTC / RTOG criteria; Grades: I - IV

I: mild, II: moderate, III-IV: severe

Patients completed a DN4 as soon as they reported moderate or severe pain.

\begin{tabular}{|c|c|c|c|c|c|c|}
\hline Variable & $\mathbf{N}(\%)$ & & & Mean (SD) & & \\
\hline & \begin{tabular}{|l} 
Total \\
$(n=26)$
\end{tabular} & $\begin{array}{l}D N 4 \geq 4 \\
(n=5)\end{array}$ & $\begin{array}{l}D N 4<4 \\
(n=21)\end{array}$ & $\begin{array}{l}\text { Total } \\
(n=26)\end{array}$ & $\begin{array}{l}D N 4 \geq 4 \\
(n=5)\end{array}$ & $\begin{array}{l}D N 4<4 \\
(n=21)\end{array}$ \\
\hline $\begin{array}{l}\text { Pain } \\
\text { Mild }\end{array}$ & |- & ( & & $7.46(1.42)$ & $8.40(0.548)$ & $7.24(1.48)$ \\
\hline $\begin{array}{l}\text { Moderate } \\
\text { Severe }\end{array}$ & $\begin{array}{l}13(50) \\
13(50)\end{array}$ & $\begin{array}{l}- \\
5(100)\end{array}$ & $\begin{array}{l}13(61.9) \\
8(38.1)\end{array}$ & & & \\
\hline Oral Mucositis & & & & $1.96(0.92)$ & $3.00(0.00)$ & $1.71(0.85)$ \\
\hline No & $3(11.5)$ & - & $3(14.3)$ & & & \\
\hline Mild & $2(7.7)$ & - & $2(9.5)$ & & & \\
\hline Moderate & $14(53.8)$ & & $14(66.7)$ & & & \\
\hline Severe & $7(26.9)$ & $5(100)$ & $2(9.5)$ & & & \\
\hline Xerostomia & & & & $6.50(2.14)$ & $8.40(0.548)$ & $6.05(2.13)$ \\
\hline Mild & $4(15.4)$ & & $4(19.0)$ & & & \\
\hline Moderate & $10(38.5)$ & - & $10(47.6)$ & & & \\
\hline Severe & $12(46.2)$ & $5(100)$ & $7(33.3)$ & & & \\
\hline
\end{tabular}

Pain, Oral Mucositis and Xerostomia in patients who completed a DN4

\begin{tabular}{|l|l|l|l|l|}
\hline NP descriptors & $\begin{array}{l}\text { Total }(\mathbf{n}=\mathbf{2 6}) \\
\mathbf{n}(\boldsymbol{\%})\end{array}$ & $\begin{array}{l}\text { Negative DN4 } \\
(\mathbf{n = 2 1}) \mathbf{n}(\boldsymbol{\%})\end{array}$ & $\begin{array}{l}\text { Positive DN4 } \\
(\mathbf{n = 5}) \mathbf{n}(\%)\end{array}$ & $\mathbf{p}$-value \\
\hline Burning & $9(34.62)$ & $6(28.6)$ & $3(60.0)$ & 0.302 \\
\hline Painful cold & - & - & - & - \\
\hline Electric shocks & $8(30.77)$ & $3(14.3)$ & $5(100)$ & $\mathbf{0 . 0 0 1}$ \\
\hline Tingling & $5(19.23)$ & $2(9.5)$ & $3(60.0)$ & $\mathbf{0 . 0 3 4}$ \\
\hline Pins and needles & $8(30.77)$ & $4(19.0)$ & $4(80.0)$ & $\mathbf{0 . 0 2 0}$ \\
\hline Numbness & $5(19.23)$ & $1(4.8)$ & $4(80.0)$ & $\mathbf{0 . 0 0 2}$ \\
\hline Itching & $4(15.38)$ & $2(9.5)$ & $2(40.0)$ & 0.155 \\
\hline Hypoesthesia to touch & $1(3.87)$ & - & $1(20.0)$ & 0.192 \\
\hline Hypoesthesia to prick & - & - & - & - \\
\hline Brushing & $9(34.62)$ & $7(33.3)$ & $2(40.0)$ & $>0.999$ \\
\hline
\end{tabular}

Statistical significance of the NP descriptors between positive $(\geq 4)$ and negative DN4 scores.

(Fisher's exact test)

Five patients (19.23\%) had a positive for NP, DN4 score $\geq 4$. The most common NP descriptor was "burning" (34.62\%) followed by "electric shocks" (30.77\%) and "pins-and-needles" (30.77\%). Statistically significant differences between positive and negative DN4q scores were observed for the "electric shocks", "tingling", "pins-and-needles" and "numbness" NP descriptors. Nine (34.62\%) patients didn't report any NP descriptors. A direct correlation was observed between DN4q score and intensity of pain, OM and xerostomia $(p<0.02)$. Pain medication was administered to fifteen (57.69\%) patients. Adjuvant medication for NP was administered to $1(1 / 5,20 \%)$ patient with positive DN4

\section{CONCLUSION:}

Oral mucositis induced NP was assessed for the first time during RT/CRT for HNC. Neuropathic pain was recorded in 5 patients with 1 of them receiving adjuvant NP medication. This study highlights the lack of adequate recognition and management of OM related NP. 